\title{
Approximation Results for Orthogonal Polynomials in Sobolev Spaces
}

\author{
By C. Canuto and A. Quarteroni
}

\begin{abstract}
We analyze the approximation properties of some interpolation operators and some $L_{\omega}^{2}$-orthogonal projection operators related to systems of polynomials which are orthonormal with respect to a weight function $\omega\left(x_{1}, \ldots, x_{d}\right), d>1$. The error estimates for the Legendre system and the Chebyshev system of the first kind are given in the norms of the Sobolev spaces $\boldsymbol{H}_{\omega}^{s}$. These results are useful in the numerical analysis of the approximation of partial differential equations by spectral methods.
\end{abstract}

0. Introduction. Spectral methods are a classical and largely used technique to solve differential equations, both theoretically and numerically. During the years they have gained new popularity in automatic computations for a wide class of physical problems (for instance in the fields of fluid and gas dynamics), due to the use of the Fast Fourier Transform algorithm.

These methods appear to be competitive with finite difference and finite element methods and they must be decisively preferred to the last ones whenever the solution is highly regular and the geometric dimension of the domain becomes large. Moreover, by these methods it is possible to control easily the solution (filtering) of those numerical problems affected by oscillation and instability phenomena.

The use of spectral and pseudo-spectral methods in computations in many fields of engineering has been matched by deeper theoretical studies; let us recall here the pioneering works by Orszag [25], [26], Kreiss and Oliger [14] and the monograph by Gottlieb and Orszag [13]. The theoretical results of such works are mainly concerned with the study of the stability of approximation of parabolic and hyperbolic equations; the solution is assumed to be infinitely differentiable, so that by an analysis of the Fourier coefficients an infinite order of convergence can be achieved. More recently (see Pasciak [27], Canuto and Quarteroni [10], [11], Maday and Quarteroni [20], [21], [22], Mercier [23]), the spectral methods have been studied by the variational techniques typical of functional analysis, to point out the dependence of the approximation error (for instance in the $L^{2}$-norm, or in the energy norm) on the regularity of the solution of continuous problems and on the discretization parameter (the dimension of the space in which the approximate solution is sought). Indeed, of ten the solution is not infinitely differentiable; on the other hand, sometimes even if the solution is smooth, its derivatives may have very

Received August 9, 1980; revised June 12, 1981.

1980 Mathematics Subject Classification. Primary 41A25; Secondary 41A10, 41A05. 
large norms which affect negatively the rate of convergence (for instance in problems with boundary layers).

Both spectral and pseudo-spectral methods are essentially Ritz-Galerkin methods (combined with some integration formulae in the pseudo-spectral case). It is well known that when Galerkin methods are used the distance between the exact and the discrete solution (approximation error) is bounded by the distance between the exact solution and its orthogonal projection upon the subspace (projection error), or by the distance between the exact solution and its interpolated polynomial at some suitable points (interpolation error). This upper bound is often realistic, in the sense that the asymptotic behavior of the approximation error is not better than the one of the projection (or even the interpolation) error. Even more, in some cases the approximate solution coincides with the projection of the true solution upon the subspace (for instance when linear problems with constant coefficients are approximated by spectral methods). This motivates the interest in evaluating the projection and the interpolation errors in differently weighted Sobolev norms. So we must face a situation different from the one of the classical approximation theory where the properties of approximation of orthogonal function systems, polynomial and trigonometric, are studied in the $L^{p}$-norms, and mostly in the maximum norm (see, e.g., Butzer and Berens [6], Butzer and Nessel [7], Nikol'skii [24], Sansone [29], Szegö [30], Triebel [31], Zygmund [32]; see also Bube [5]). Approximation results in Sobolev norms for the trigonometric system have been obtained by Kreiss and Oliger [15]. In this paper we consider the systems of Legendre orthogonal polynomials, and of Chebyshev orthogonal polynomials of the first kind in dimension $d \geqslant 1$. The reason for this interest must be sought in the applications to spectral approximations of boundary value problems. Indeed, if the boundary conditions are not periodic, Legendre approximation seems to be the easiest to be investigated (the weight $\omega$ is equal to 1). On the other hand, the Chebyshev approximation is the most effective for practical computations since it allows the use of the Fast Fourier Transform algorithm.

The techniques used to obtain our results are based on the representation of a function in the terms of a series of orthogonal polynomials, on the use of the so-called inverse inequality, and finally on the operator interpolation theory in Banach spaces. For the theory of interpolation we refer for instance to Calderon [8], Lions [17], Lions and Peetre [19], Peetre [28]; a recent survey is given, e.g., by Bergh and Löfström [4].

An outline of the paper is as follows. In Section 1 some approximation results for the trigonometric system are recalled; the presentation of the results to the interpolation is made in the spirit of what will be its application to Chebyshev polynomials.

In Section 2 we consider the $L_{\omega}^{2}$-projection operator upon the space of polynomials of degree at most $N$ in any variable ( $\omega$ denotes the Chebyshev or Legendre weight). In Section 3 a general interpolation operator, built up starting by integration formulas which are not necessarily the same in different spatial dimensions, is considered, and its approximation properties are studied.

In [22] Maday and Quarteroni use the results of Section 2 to study the approximation properties of some projection operators in higher order Sobolev norms. Recently, an interesting method which lies inbetween finite elements and 
spectral methods has been investigated from the theoretical point of view by Babuška, Szabö and Katz [3]. In particular they obtain approximation properties of polynomials in the norms of the usual Sobolev spaces.

Acknowledgements. Some of the results of this paper were announced in [9]; we thank Professor J. L. Lions for the presentation to the C. R. Acad. Sci. of Paris. We also wish to express our gratitude to Professors F. Brezzi and P. A. Raviart for helpful suggestions and continuous encouragement.

Notations. Throughout this paper we shall use the following notations: $I$ will be an open bounded interval $\subset \mathbf{R}$, whose variable is denoted by $x ; \Omega$ the product $I^{d} \subset \mathbf{R}^{d}(d$ integer $\geqslant 1)$ whose variable is denoted by $\mathbf{x}=\left(x^{(j)}\right)_{j-1, \ldots, d}$; for a multi-integer $\mathbf{k} \in \mathbf{Z}^{d}$, we set $|\mathbf{k}|^{2}=\sum_{j=1}^{d}\left|k_{j}\right|^{2}$ and $|\mathbf{k}|_{\infty}=\max _{1<j<d}\left|k_{j}\right|$. When $d=1$ we set $D=d / d x$, when $d>1, D_{j}=\partial / \partial x^{(j)}$. The symbol $\Sigma^{\prime} q_{k=p}(q$ eventually $+\infty$ ) will denote the summation over all integral $k$ such that $p<k \leqslant q$ and $k-p$ is even.

We use weighted Sobolev spaces of Hilbert type over the open set $\Omega$ : we recall here some definitions (see, e.g., Adams [1], Avantaggiati [2], Kufner, John and Fucik [16], Lions and Magenes [18]). We are given a weight function $\omega$ on $\Omega$ satisfying $\omega \in L^{1}(\Omega), \omega(\mathbf{x})>0$ in $\Omega$. Set

$$
\mathbf{L}_{\omega}^{2}(\Omega)=\left\{\phi: \Omega \rightarrow \mathbf{C} \mid \phi \text { is measurable and }(\phi, \phi)_{\omega}<+\infty\right\}
$$

equipped with the inner product

$$
(\phi, \psi)_{\omega}=\int_{\Omega} \phi(\mathbf{x}) \overline{\psi(\mathbf{x})} \omega(\mathbf{x}) d \mathbf{x} .
$$

For any integral $s \geqslant 0$, set

$$
\mathbf{H}_{\omega}^{s}(\Omega)=\left\{\phi \in \mathbf{L}_{\omega}^{2}(\Omega) \mid\|\phi\|_{s, \omega}<+\infty\right\}
$$

where

$$
\|\phi\|_{s, \omega}^{2}=\sum_{\substack{\mathbf{k} \in \mathbf{N}^{d} \\ k_{1}+k_{2}+\cdots+k_{d}<s}} \int_{\Omega}\left|\left(\prod_{j=1}^{d} D_{j}^{k_{j}}\right) \phi\right|^{2} \omega d \mathbf{x} .
$$

For real $s>0$, define $\mathbf{H}_{\omega}^{s}(\Omega)$ by complex interpolation between $\mathbf{H}_{\omega}^{\bar{s}}(\Omega)$ and $\mathbf{H}_{\omega}^{\bar{s}+1}(\Omega)$, where $\bar{s}$ denotes the largest integer smaller than $s$ (see, e.g., Bergh and Löfström [4]).

Finally, $L_{\omega}^{2}(\Omega)$ and $H_{\omega}^{s}(\Omega)$ will denote the subspaces of $\mathbf{L}_{\omega}^{2}(\Omega)$ and $\mathbf{H}_{\omega}^{s}(\Omega)$ of real-valued functions; if $\omega \equiv 1$, the subscript $\omega$ will be systematically dropped.

1. The Fourier System. In this section only, we assume $I=\tilde{I}=(-\pi, \pi)$, and we set $\tilde{\Omega}=\tilde{I}^{d}$; its variables will be denoted by $\boldsymbol{\theta}, \boldsymbol{\theta}$ instead of $x, \mathbf{x}$. We consider the set $\left\{\psi_{\mathbf{k}} \mid \mathbf{k} \in \mathbf{Z}^{d}\right\}$, with $\psi_{\mathbf{k}}(\boldsymbol{\theta})=(2 \pi)^{-d / 2} \exp (i \mathbf{k} \cdot \boldsymbol{\theta})$, which forms a complete orthonormal system in $\mathbf{L}^{2}(\tilde{\Omega})$. Given $C_{(p)}^{\infty}(\tilde{\Omega})=\left\{v=V_{\mid \tilde{\Omega}} \mid V: \mathbf{R}^{d} \rightarrow \mathbf{C}\right.$ is infinitely differentiable and $2 \pi$-periodic in each variable $\}$, we define for any $s \geqslant 0 \mathbf{H}_{(p)}^{s}(\tilde{\Omega})=$ closure of $C_{(p)}^{\infty}(\tilde{\Omega})$ in $\mathbf{H}^{s}(\tilde{\Omega})$. Since $D_{j} \psi_{\mathbf{k}}=i k_{j} \psi_{\mathbf{k}}$, for any $u=\Sigma_{\mathbf{k} \in \mathbf{Z}^{d}} \hat{u}_{\mathbf{k}} \psi_{\mathbf{k}}$ the norm $\|u\|_{s}=$ $\left(|u|_{0}^{2}+|u|_{s}^{2}\right)^{1 / 2}$, where $|u|_{s}^{2}=\Sigma_{\mathbf{k} \in \mathbf{Z}^{d}}|\mathbf{k}|^{2 s}\left|\hat{u}_{\mathbf{k}}\right|^{2}$, is equivalent to the $\mathbf{H}^{s}(\tilde{\Omega})$-norm $\|u\|_{s}$ (see, e.g., Pasciak [27]). For any positive integer $N$, set

$$
\tilde{S}_{N}=\operatorname{span}\left\{\left.\psi_{\mathbf{k}}|| \mathbf{k}\right|_{\infty} \leqslant N\right\} \subset \mathbf{L}^{2}(\Omega),
$$


and denote by $\tilde{P}_{N}: \mathbf{L}^{2}(\tilde{\Omega}) \rightarrow \tilde{S}_{N}$ the orthogonal projection operator. Note that $\tilde{P}_{N}$ commutes with derivation, i.e., $\tilde{P}_{N} D_{j}=D_{j} \tilde{P}_{N}, 1 \leqslant j \leqslant d$.

THEOREM 1.1. For any real $0 \leqslant \mu \leqslant \sigma$, there exists a constant $C$ such that

$$
\left\|u-\tilde{P}_{N} u\right\|_{\mu} \leqslant C N^{\mu-\sigma}|u|_{\sigma} \quad \forall u \in \mathbf{H}_{(p)}^{o}(\tilde{\Omega})
$$

Proof. One has

$$
\begin{aligned}
\left\|u-\tilde{P}_{N} u \mid\right\|_{\mu} & =\sum_{|\mathbf{k}|_{\infty}>N}\left(1+|\mathbf{k}|^{2 \mu}\right)\left|\hat{u}_{\mathbf{k}}\right|^{2} \leqslant 2 \sum_{|\mathbf{k}|_{\infty}>N}|\mathbf{k}|^{2 \sigma-2(\sigma-\mu)}\left|\hat{u}_{\mathbf{k}}\right|^{2} \\
& \leqslant 2 N^{2(\mu-\sigma)}|u|_{\sigma}^{2} . \square
\end{aligned}
$$

Moreover, the following inverse inequality can be easily checked:

Proposition 1.1. For any real $0 \leqslant \nu \leqslant \mu$,

$$
|\phi|_{\mu} \leqslant N^{\mu-\nu}|\phi|_{\nu} \quad \forall \phi \in \tilde{S}_{N}
$$

and hence

$$
\|\phi\|_{\mu} \leqslant C N^{\mu-\nu}\|\phi\|_{\nu} \quad \forall \phi \in \tilde{S}_{N}
$$

We now construct some trigonometric interpolation operators over $\tilde{\Omega}$. The set of interpolation points will be the cartesian product of $d$ (possibly different) sets of interpolation points over $\tilde{I}$. So, we start by introducing three different interpolation operators in one dimension. The corresponding interpolation points are equispaced over $\tilde{I}$, so they will give rise to the Chebyshev points over $[-1,1]$ (see Section 3.1). We call them of type $(G),(G R)$ or $(G L)$, since they are associated with the integration formulae of Gauss, Gauss-Radau or Gauss-Lobatto, respectively, with respect to the Chebyshev weight. This material will be used in Section 3.

We consider interpolation points of the form

$$
\theta_{m}=\theta_{0}+m h \in \tilde{I}, \quad m=0, \ldots, M, \quad h=2 \pi /(M+1)
$$

for given $\theta_{0} \in \tilde{I}$ and $M \in \mathrm{N}$. We associate to them a bilinear form on $C^{0}([-\pi, \pi])$

$$
(u, v)_{N}=h \sum_{m=0}^{M} u\left(\theta_{m}\right) \overline{v\left(\theta_{m}\right)}
$$

and an interpolation operator at the points $\theta_{m}, m=0, \ldots, M$,

$$
\tilde{\Pi}_{c}: C^{0}([-\pi, \pi]) \rightarrow \tilde{\Xi}_{N}
$$

over a space of dimension $M+1$ spanned by given trigonometric polynomials. The following identities will be used:

$$
\frac{1}{M+1} \sum_{m=0}^{M+1} \exp (\text { ihpm })= \begin{cases}1 & \text { if } p \in(M+1) Z \\ 0 & \text { otherwise. }\end{cases}
$$

(a) Interpolation Points of Type $(G)$. We choose

$$
\theta_{0}=-\frac{2 N+1}{2 N+2} \pi \text { and } M=2 N+1
$$

By (1.7) we get

$$
\left(\psi_{l}, \psi_{k}\right)_{N}= \begin{cases}(-1)^{q} & \text { if } l-k=2 q(N+1), q \in \mathbf{Z} \\ 0 & \text { otherwise }\end{cases}
$$


Hence if $\tilde{\Xi}_{N}=\tilde{\Xi}_{N}^{(G)}=\left\{\eta \in \tilde{S}_{N+1}(\tilde{I}) \mid \hat{\eta}_{-(N+1)}=0\right\}$, one has

(1.10) $\tilde{\Pi}_{c} v=\sum_{k=-N}^{N+1} \tilde{v}_{k} \psi_{k} \quad$ with $\tilde{v}_{k}=\left(v, \psi_{k}\right)_{N}=\sum_{q=-\infty}^{+\infty}(-1)^{q} \hat{v}_{k+2 q(N+1)}$.

(b) Interpolation Points of Type (GR). We choose

$$
\theta_{0}=-\pi \text { and } M=2 N \text {. }
$$

By (1.7) we get

$$
\left(\psi_{l}, \psi_{k}\right)_{N}= \begin{cases}(-1)^{q} & \text { if } l-k=(2 N+1) q, q \in \mathbf{Z} \\ 0 & \text { otherwise. }\end{cases}
$$

hence if $\tilde{\Xi}_{N}=\tilde{\Xi}_{N}^{(G R)}=\tilde{S}_{N}$, one has

(1.13) $\tilde{\Pi}_{c} v=\sum_{k=-N}^{N} \tilde{v}_{k} \psi_{k} \quad$ with $\tilde{v}_{k}=\left(v, \psi_{k}\right)_{N}=\sum_{q=-\infty}^{\infty}(-1)^{q} \hat{v}_{k+(2 N+1) q}$

(c) Interpolation Points of Type (GL). We choose

$$
\theta_{0}=-\pi \text { and } M=2 N-1 .
$$

By (1.7) we get

$$
\left(\psi_{l}, \psi_{k}\right)_{N}= \begin{cases}1 & \text { if } l-k=2 N q, q \in \mathbf{Z} \\ 0 & \text { otherwise. }\end{cases}
$$

Note that

$$
\left(v, \psi_{N}\right)_{N}=\left(v, \psi_{-N}\right)_{N} \quad \forall v \in C^{0}([-\pi, \pi])
$$

Hence if $\tilde{\Xi}_{N}=\tilde{\Xi}_{N}^{(G L)}=\left\{\psi \in \tilde{S}_{N} \mid \hat{\psi}_{N}=\hat{\psi}_{-N}\right\}$,

$$
\tilde{\Pi}_{c} v=\sum_{k=-N}^{N} \tilde{v}_{k} \psi_{k} \quad \text { with } \tilde{v}_{k}=\left(v, \psi_{k}\right)_{N}=\sum_{q=-\infty}^{\infty} \hat{v}_{k+2 q N}
$$

where the asterisk means that $\tilde{v}_{N}$ and $\tilde{v}_{-N}$ must be multiplied by $1 / 2$.

We now consider the general dimension $d$. We divide the set $\mathscr{D}=\{1, \ldots, d\}$ into three disjoint (eventually void) sets $J(G), J(G R), J(G L)$; then we put

$$
\nu=\left(\nu_{j}\right) \in \mathbf{N}^{d}, \quad \nu_{j}= \begin{cases}2 N+2 & \text { if } j \in J(G), \\ 2 N+1 & \text { if } j \in J(G R), \\ 2 N & \text { if } j \in J(G L),\end{cases}
$$

and we define the sets

$$
\begin{aligned}
& \xi(N)=\left\{\mathbf{m} \in \mathbf{N}^{d} \mid m_{j} \leqslant \nu_{j}-1, \forall j \in \mathscr{D}\right\}, \\
& \zeta(N)=\left\{\mathbf{k} \in \mathbf{Z}^{d} \mid-N \leqslant k_{j} \leqslant \begin{cases}N+1 & \text { if } j \in J(G), \\
N & \text { if } j \in J(G R) \cup J(G L),\end{cases} \right.
\end{aligned}
$$

and the interpolation points

$$
\begin{aligned}
\boldsymbol{\theta}_{\mathbf{m}}=\left(\theta_{m_{j}}^{(j)}\right), \quad \mathbf{m} \in \xi(N), & \text { where } \theta_{m_{j}}^{(j)}=\theta_{m_{j}} \\
& \text { defined in (a), (b) or (c) according as } j \in J(G), \\
& j \in J(G R) \text { or } j \in J(G L) .
\end{aligned}
$$

We can now define the following bilinear form on $C^{0}(\overline{\tilde{\Omega}})$

$$
(u, v)_{N}=c_{N} \sum_{\mathbf{m} \in \xi(N)} u\left(\theta_{\mathrm{m}}\right) \overline{v\left(\theta_{\mathrm{m}}\right)}
$$

with $c_{N}=\left(h^{(G)}\right)^{\text {card } J(G)} \times\left(h^{(G R)}\right)^{\text {card } J(G R)} \times\left(h^{(G L)}\right)^{\text {card } J(G L)}$. 
Finally we set

$$
\tilde{\Xi}_{N}(\tilde{\Omega})=\left\{\eta=\sum_{\mathbf{k} \in \zeta(N)} \hat{\eta}_{\mathbf{k}} \psi_{\mathbf{k}} \mid \hat{\eta}_{\mathbf{k}}=\hat{\eta}_{\mathbf{l}} \text { whenever } \mathbf{k} \text { and } \mathbf{l}\right. \text { differ at }
$$
most in the sign of the components $k_{j}, l_{j}$ with

$$
\left.j \in J(G L) \text { and }\left|k_{j}\right|=\left|l_{j}\right|=N\right\}
$$

(so that $\tilde{\Xi}_{N}(\tilde{\Omega}) \simeq \Pi_{j \in J(G)} \tilde{\Xi}_{N}^{(G)} \times \prod_{j \in J(G R)} \tilde{S}_{N} \times \prod_{j \in J(G L)} \tilde{\Xi}_{N}^{(G L)}$ ), and we define the interpolation operator $\tilde{\Pi}_{c}: C^{0}(\overline{\tilde{\Omega}}) \rightarrow \tilde{\Xi}_{N}(\tilde{\Omega})$ by the relation

$$
\left(\tilde{\Pi}_{c} v, \psi\right)_{N}=(v, \psi)_{N}, \quad \forall \psi \in \tilde{\Xi}_{N} .
$$

We agree that $\sum_{\mathbf{k} \in \zeta(N)}^{*} \alpha_{\mathbf{k}}$ means that in summing every term $\alpha_{\mathbf{k}}$ must be multiplied by $\frac{1}{2}$ raised to the number of components $k_{j}$ in $\mathbf{k}$ with $j \in J(G L)$ and $\left|k_{j}\right|=N$. Then, if $\tilde{\Pi}_{c} v=\sum_{\mathbf{k} \in \zeta(N)}^{*} \tilde{v}_{\mathbf{k}} \psi_{\mathbf{k}}$, one obtains, recalling (1.16),

$$
\tilde{v}_{\mathbf{k}}=\left(v, \psi_{\mathbf{k}}\right)_{N} \quad \forall \mathbf{k} \in \zeta(N)
$$

$\tilde{\Pi}_{c} v$ is equal to $v$ at the nodes (1.19) since, by (1.2) and (1.7), one has

$$
\begin{aligned}
\tilde{\Pi}_{c} v\left(\theta_{\mathbf{m}}\right) & =\sum_{\mathbf{k} \in \zeta(N)} *\left[c_{N} \sum_{\mathbf{n} \in \xi(N)} v\left(\theta_{\mathbf{n}}\right) \psi_{\mathbf{k}}\left(-\boldsymbol{\theta}_{\mathbf{n}}\right)\right] \psi_{\mathbf{k}}\left(\boldsymbol{\theta}_{\mathbf{m}}\right) \\
& =\sum_{\mathbf{n} \in \xi(N)} v\left(\boldsymbol{\theta}_{\mathbf{n}}\right)\left[c_{N} \sum_{\mathbf{k} \in \zeta(N)}^{*} \psi_{\mathbf{k}}\left(-\boldsymbol{\theta}_{\mathbf{n}}\right) \psi_{\mathbf{k}}\left(\boldsymbol{\theta}_{\mathbf{m}}\right)\right] \\
& =\sum_{\mathbf{n} \in \xi(N)} v\left(\boldsymbol{\theta}_{\mathbf{n}}\right) \delta_{\mathbf{n}, \mathbf{m}} .
\end{aligned}
$$

THEOREM 1.2. For any real $0 \leqslant \mu \leqslant \sigma$ with $\sigma>d / 2$, there exists a constant $C$ such that

$$
\left\|u-\tilde{\Pi}_{c} u\right\|_{\mu} \leqslant C N^{\mu-\sigma}|u|_{\sigma} \quad \forall u \in \mathbf{H}_{(p)}^{\sigma}(\tilde{\Omega}) .
$$

Proof. The theorem can be proved following the technique used by Pasciak in [27, Proof of Theorem 3], where only points of type $(G L)$ are considered.

2. Spectral Projection Systems. We consider a weight function $w \in L^{1}(I), w>0$ in $I$, and for any $d \geqslant 1$ we define

$$
\omega(\mathbf{x})=\prod_{j=1}^{d} w\left(x^{(j)}\right) .
$$

If $\left\{\phi_{k}\right\}_{k=0}^{\infty}$ is the system of the orthogonal polynomials in $L_{w}^{2}(I)$-with $\operatorname{deg} \phi_{k}=k$ -(see e.g. Szegö [30]), then the system

$$
\left\{\phi_{\mathbf{k}}\right\}_{\mathbf{k} \in \mathbf{N}^{d}}, \quad \text { where } \phi_{\mathbf{k}}(\mathbf{x})=\prod_{j=1}^{d} \phi_{k_{j}}\left(x^{(j)}\right),
$$

is orthonormal and complete in $L_{\omega}^{2}(\Omega)$; any $v \in L_{\omega}^{2}(\Omega)$ can be written as

$$
v=\sum_{\mathbf{k} \in \mathbf{N}^{d}} \hat{v}_{\mathbf{k}} \phi_{\mathbf{k}}, \quad \hat{v}_{\mathbf{k}}=\left(v, \phi_{\mathbf{k}}\right)_{\omega}
$$

with

$$
\|v\|_{0, \omega}^{2}=\sum_{k \in N^{d}}\left|\hat{v}_{\mathbf{k}}\right|^{2}
$$


Setting

$$
S_{N}=S_{N}(\Omega)=\operatorname{span}\left\{\left.\phi_{\mathbf{k}}\left|\mathbf{k} \in \mathbf{N}^{d},\right| \mathbf{k}\right|_{\infty}<N\right\}
$$

( $S_{N}$ is the set of all polynomials of degree $\leqslant N$ in each variable), we denote by $P_{N}$ : $L_{\omega}^{2}(\Omega) \rightarrow S_{N}$ the orthogonal projection on $S_{N}$ in $L_{\omega}^{2}(\Omega)$.

Definition 2.1. The triplet $\left(\Omega, \omega, P_{N}\right)$ is called a Spectral Projection System (SPS).

2.1. The Chebyshev SPS. We choose $I=(-1,1)$ and $w(x)=\left(1-x^{2}\right)^{-1 / 2}$. If $T_{k}$ denotes the Chebyshev polynomial of the first kind of degree $k, T_{k}(\cos \theta)=$ $\cos k \theta,\left\{\phi_{k}=\tau_{k} T_{k}\right\}_{k=0}^{\infty}$ (with $\tau_{0}=\pi^{-1 / 2}, \tau_{k}=(2 / \pi)^{1 / 2}$ for $k>1$ ) is the orthonormal system associated with the weight $w$.

We associate to every function $v=v(\mathbf{x})$ on $\Omega$ the function

$$
\tilde{v}(\theta)=v(\cos \theta), \quad \text { where } \cos \theta=\left(\cos \theta^{(1)}, \cos \theta^{(2)}, \ldots, \cos \theta^{(d)}\right)
$$

as an even, periodic function on $\tilde{\Omega}$. Since $\int_{\Omega} v(\mathbf{x}) \omega(\mathbf{x}) d \mathbf{x}=2^{-d} \int_{\tilde{\Omega}} \tilde{v}(\boldsymbol{\theta}) d \boldsymbol{\theta}$ and $d \theta / d x=w \geqslant 1$, it is easily checked that

(2.7) the mapping $v \rightarrow \tilde{v}$ is a continuous injection of $H_{\omega}^{s}(\Omega)$ into $H_{(p)}^{s}(\tilde{\Omega})$.

Clearly it also maps $S_{N}$ into $\tilde{S}_{N}$ : more precisely for any $\mathbf{k} \in \mathbf{N}^{d}, \tilde{\boldsymbol{\phi}}_{\mathbf{k}} \in$ $\operatorname{span}\left\{\psi_{\mathbf{l}} \mid \mathbf{l} \in \mathbf{Z}^{d}\right.$ and $\left.\left|l_{j}\right|=k_{j}, 1 \leqslant j \leqslant d\right\}$. In particular, this implies that

$$
\widetilde{P_{N} v}=\tilde{P}_{N} \tilde{v} \quad \forall v \in L_{\omega}^{2}(\Omega) \text {. }
$$

We can now derive the rates of convergence (with respect to $N$ ) for the approximation error $u-P_{N} u$ in the Sobolev norms. The estimate in the $L_{\omega}^{2}$-norm is an easy consequence of Proposition 1.1.

THEOREM 2.1. For any real $\sigma \geqslant 0$, there exists a constant $C$ such that

$$
\left\|u-P_{N} u\right\|_{0, \omega} \leqslant C N^{-\sigma}\|u\|_{\sigma, \omega} \quad \forall u \in H_{\omega}^{\sigma}(\Omega)
$$

Proof. One has

$$
\begin{aligned}
\left\|u-P_{N} u\right\|_{0, \omega} & =2^{-d / 2}\left\|\tilde{u}-\tilde{P}_{N} \tilde{u}\right\|_{\mathbf{L}^{2}(\tilde{\Omega})} & & \text { by }(2.8) \\
& \leqslant 2^{-d / 2} \tilde{C} N^{-\sigma}|\tilde{u}|_{\sigma} & & \text { by }(1.1) \\
& \leqslant C N^{-\sigma}\|u\|_{\sigma, \omega} & & \text { by }(2.7)
\end{aligned}
$$

We now consider the Sobolev norms of higher order. To this end, let us recall that the following formal expansion of the derivative of a function $v=\sum_{k=0}^{\infty} \hat{v}_{k} \phi_{k}$ holds:

$$
D v=\sum_{l=0}^{\infty} \gamma_{l}\left(\sum_{k=l+1}^{\infty} k \hat{v}_{k}\right) \phi_{l}, \quad \gamma_{0}=\sqrt{2}, \gamma_{l}=1, \text { for } l \geqslant 1 .
$$

This expansion can be rigorously justified whenever $v$ is regular enough; for instance if $\left|\hat{v}_{k}\right|=O\left(k^{-3}\right)$ for $k \rightarrow \infty$, the right-hand side of (2.10) defines a $L_{\omega}^{2}(I)$-function which is the distributional derivative of $v$ (see the proof of Lemma 2.2 below). The following inverse inequality will be used.

LEMMA 2.1. For any real $\mu$ and $\nu$ such that $0 \leqslant \nu \leqslant \mu$, there exists a constant $C$ such that

$$
\|u\|_{\mu, \omega} \leqslant C N^{2(\mu-\nu)}\|u\|_{\nu, \omega} \quad \forall u \in S_{N}
$$


Proof. For $v=\sum_{|\mathbf{k}|_{\infty} \leqslant N} \hat{v}_{\mathbf{k}} \phi_{\mathbf{k}}$ one has by (2.10)

$$
D_{1} v=\sum_{\|\|_{\infty}<N} \gamma_{l_{1}}\left(\sum_{k_{1}=l_{1}+1}^{N} k_{1} \hat{v}_{\left(k_{1}, \mathrm{I}^{\prime}\right)}\right) \phi_{1}
$$

where $\mathbf{l}^{\prime}=\left(l_{2}, \ldots, l_{d}\right)$. Hence

$$
\begin{aligned}
\left\|D_{1} v\right\|_{0, \omega}^{2} & =\sum_{\substack{||_{\infty}<N \\
l_{1}<N}} \gamma_{l_{1}}^{2}\left(\sum_{k_{1}=l_{1}+1}^{N} k_{1} \hat{v}_{\left(k_{1}, l^{\prime}\right)}\right)^{2} \\
& \leqslant 2 \sum_{l_{1}=0}^{N-1} \sum_{\left.||^{\prime}\right|_{\infty}<N}\left(\sum_{k_{1}=l_{1}+1}^{N} k_{1}^{2}\right) \cdot\left(\sum_{k_{1}=l_{1}+1}^{N}\left|\hat{v}_{\left(k_{1}, l^{\prime}\right)}\right|^{2}\right) \\
& \leqslant \frac{N(N+1)(2 N+3)}{3} \sum_{l_{1}=0}^{N-1} \sum_{k_{1}=1}^{N} \sum_{\left|I^{\prime}\right|_{\infty}<N}\left|\hat{v}_{\left(k_{1}, l^{\prime}\right)}\right|^{2} \\
& \leqslant C N^{4}\|v\|_{0, \omega}^{2},
\end{aligned}
$$

and similarly for the other first-order derivatives of $v$. A repeated application of these estimates yields

$$
\|u\|_{m, \omega} \leqslant C N^{2 m}\|u\|_{0, \omega} \quad \forall u \in S_{N}
$$

for any integral $m$. For real $\mu$ we use interpolation of spaces: let $S_{N}^{(\sigma)}$ denote the space $S_{N}$ equipped with the $H_{\omega}^{\sigma}$-norm, then the identity $i: S_{N}^{(0)} \rightarrow S_{N}^{(m)}$ has norm $<C N^{2 m}$, while obviously $i: S_{N}^{(0)} \rightarrow S_{N}^{(0)}$ has norm $=1$. By Bergh and Löfström [4], Theorems 4.1.2 and 4.2.1(c), we get that $i: S_{N}^{(0)} \rightarrow\left(S_{N}^{(m)}, S_{N}^{(0)}\right)_{\{\theta]}=S_{N}^{(\theta m)}$ has norm $<C N^{2 \theta m}, 0<\theta<1$. Finally, for nonzero $\nu$, we interpolate between $i: S_{N}^{(0)} \rightarrow S_{N}^{(\mu)}$ and $i: S_{N}^{(\mu)} \rightarrow S_{N}^{(\mu)}$ and again use Theorem 4.1.2 quoted above.

Remark 2.1. The bound of the quantity $\left\|D_{1} v\right\|_{0, \omega}$ in the proof of the lemma may seem quite crude. Nevertheless the power of $N$ in estimate (2.11) is optimal, in the sense that it cannot be reduced. Actually, for $d=1$, consider the element

$$
u=\sum_{k=0}^{N} \phi_{k}, \quad N \text { even, }
$$

for which one has

$$
D u=\sum_{l=1}^{N-1} \gamma_{l}\left(\sum_{k=l+1}^{N} k\right) \phi_{l}, \quad D^{2} u=\sum_{l=0}^{N-2} \gamma_{l}\left(\sum_{k=l+2}^{N} k\left(k^{2}-l^{2}\right)\right) \phi_{l}
$$

Then one easily checks that

$$
\|u\|_{j, \omega} \simeq N^{2 j}\|u\|_{0, \omega}, \quad j=1,2
$$

The formal expansion of the first derivative of a function, given in (2.10), shows that $P_{N}$ cannot commute with derivation, as for the Fourier system. Therefore, we need bounds for the Sobolev norms of the commutators $P_{N} D_{j}-D_{j} P_{N}(j=$ $1, \ldots, d)$, which we shall establish in the following lemmas. For the sake of simplicity, we only deal with the case $j=1$, the extension to an arbitrary $j$ being obvious. 
LeMma 2.2. Let $u \in H_{\omega}^{1}(\Omega)$, and set $D_{1} u=\sum_{\mathbf{k} \in N^{d}} \hat{z}_{\mathbf{k}} \phi_{\mathbf{k}}$. Then

$$
P_{N} D_{1} u-D_{1} P_{N} u= \begin{cases}z^{(N)} \cdot \phi_{0}^{(N)}+z^{(N+1)} \cdot \phi_{1}^{(N)}, & N \text { even, } \\ z^{(N)} \cdot \phi_{I}^{(N)}+z^{(N+1)} \phi_{0}^{(N)}, & N \text { odd },\end{cases}
$$

where

$$
\begin{array}{cc}
z^{(N)}=\sum_{\substack{\mathbf{k}^{\prime} \in N^{d-1} \\
\left|\mathbf{k}^{\prime}\right|_{\infty}<N}} \hat{z}_{\left(N, \mathbf{k}^{\prime}\right)} \phi_{\mathbf{k}^{\prime}}, & z^{(N+1)}=\sum_{\substack{\mathbf{k}^{\prime} \in N^{d-1} \\
\left|\mathbf{k}^{\prime}\right|_{\infty}<N}} \hat{z}_{\left(N+1, \mathbf{k}^{\prime}\right)} \phi_{\mathbf{k}^{\prime}}, \\
\phi_{0}^{(N)}=\sum_{l_{1}=0}^{N} \gamma_{l_{1}} \phi_{l_{1}}, & \phi_{1}^{(N)}=\sum_{l_{1}=1}^{N} \gamma_{l_{1}} \phi_{l_{1}} .
\end{array}
$$

Proof. We assume at first that $u$ is regular enough, so that one has, by (2.10),

$$
\hat{z}_{\mathbf{k}}=\gamma_{k_{1}} \cdot \sum_{m=k_{1}+1}^{\infty} m \hat{u}_{\left(m, \mathbf{k}^{\prime}\right)}
$$

where $\mathbf{k}^{\prime}=\left(k_{2}, \ldots, k_{d}\right)$. For instance, since by (2.9) one gets

$$
\left|\hat{u}_{1}\right| \leqslant\left\|u-P_{L} u\right\|_{0, \omega} \leqslant C L^{-\sigma}\|u\|_{\sigma, \omega}, \quad L=\mid \|_{\infty}-1
$$

for any $l \in \mathrm{N}^{d}$ and any $u \in H_{\omega}^{\sigma}(\Omega)$, one can take $\sigma$ so large that the series on the right-hand side of (2.14) is absolutely convergent, and equals the kth Chebyshev coefficient of a function $z \in L_{\omega}^{2}(\Omega)$, which necessarily coincides with $D_{1} u$. Then

$$
\begin{aligned}
& P_{N} D_{1} u=\sum_{\substack{\mathbf{k}^{\prime} \in \mathbf{N}^{d-1} \\
\left|\mathbf{k}^{\prime}\right|<N}} \phi_{\mathbf{k}^{\prime}} \sum_{l_{1}=0}^{N} \gamma_{l_{1}}\left(\sum_{k_{1}=l_{1}+1}^{\infty} k_{1} \hat{u}_{\left(k_{1}, \mathbf{k}^{\prime}\right)}\right) \phi_{l_{1}}, \\
& D_{1} P_{N} u=\sum_{\substack{\mathbf{k}^{\prime} \in \mathbf{N}^{d-1} \\
\left|\mathbf{k}^{\prime}\right|<N}} \phi_{\mathbf{k}^{\prime}} \sum_{l_{1}=0}^{N-1} \gamma_{l_{1}}\left(\sum_{k_{1}=l_{1}+1}^{N} k_{1} \hat{u}_{\left(k_{1}, \mathbf{k}^{\prime}\right)}\right) \phi_{l_{1}},
\end{aligned}
$$

whence (2.13). Since each side of (2.13) belongs to the finite dimensional subspace $S_{N}$ and depends continuously on $u$ in the topology of $H_{\omega}^{1}(\Omega)$, a standard density argument yields the result for arbitrary $u \in H_{\omega}^{1}(\Omega)$.

LEMMA 2.3. For any real $\rho$ and $\sigma$ with $0 \leqslant \rho<\sigma-1$ there exists a constant $C$ such that for $j=1, \ldots, d$,

$$
\left\|\left(P_{N} D_{j}-D_{j} P_{N}\right) u\right\|_{\rho, \omega} \leqslant C N^{2 \rho-\sigma+3 / 2}\|u\|_{\sigma, \omega} \quad \forall u \in H_{\omega}^{\sigma}(\Omega) .
$$

Proof. Again we may consider only the case $j=1$. We remark that $z^{(N)}, z^{(N+1)}$ only depend on $\mathbf{x}^{\prime}=\left(x_{2}, \ldots, x_{d}\right)$ and belong to the subspace $S_{N}$ (in $(d-1)$ dimensions). Conversely $\phi_{0}^{(N)}, \phi_{1}^{(N)}$ only depend on $x_{1}$ and are orthogonal in $L_{w}^{2}(I)$. Hence, when $N$ is even

$$
\left\|P_{N} D_{1} u-D_{1} P_{N} u\right\|_{0, \omega}^{2}=\left\|z^{(N)}\right\|_{0, \omega^{\prime}}^{2}\left\|\phi_{0}^{(N)}\right\|_{0, w}^{2}+\left\|z^{(N+1)}\right\|_{0, \omega^{\prime}}^{2}\left\|\phi_{1}^{(N)}\right\|_{0, w}^{2}
$$

and analogously when $N$ is odd.

By (2.9) we get

$$
\left\|z^{(N)}\right\|_{0, \omega^{\prime}}^{2}=\sum_{\substack{\mathbf{k}^{\prime} \in \mathbf{N}^{d-1} \\|\mathbf{k}|_{\infty}<N}}\left|\hat{z}_{\left(N, \mathbf{k}^{\prime}\right)}\right|^{2} \leqslant\left\|D_{1} u-P_{N-1} D_{1} u\right\|_{0, \omega}^{2} \leqslant C N^{2(1-s)}\|u\|_{s, \omega}^{2},
$$


and the same bound holds for $\left\|z^{(N+1)}\right\|_{0, \omega^{\prime}}^{2}$; since

$$
\left\|\phi_{0}^{(N)}\right\|_{0, w}^{2} \sim N, \quad\left\|\phi_{1}^{(N)}\right\|_{0, w}^{2} \sim N
$$

we obtain (2.15) for $\rho=0$. For $\rho>0$ we conclude by the inverse inequality (2.11).

Now, we are able to state the main estimate for the approximation error $u-P_{N} u$.

THEOREM 2.2. For any real $\mu$ and $\sigma$ such that $0 \leqslant \mu \leqslant \sigma$, there exists a constant $C$ such that

$$
\left\|u-P_{N} u\right\|_{\mu, \omega} \leqslant C N^{e(\mu, \sigma)}\|u\|_{\sigma, \omega} \quad \forall u \in H_{\omega}^{\sigma}(\Omega)
$$

where

$$
e(\mu, \sigma)= \begin{cases}2 \mu-\sigma-1 / 2, & \mu \geqslant 1, \\ 3 \mu / 2-\sigma, & 0 \leqslant \mu \leqslant 1 .\end{cases}
$$

Proof. It is enough to prove the result for the integral $\mu$, and then use interpolation; see Bergh and Löfström [4]. If $\mu=0,(2.16)$ reduces to (2.10); so assume by inductive hypothesis that (2.16) holds for any integer $\mu \leqslant m-1$. One has

$$
\begin{aligned}
\left\|u-P_{N} u\right\|_{m, \omega} & \leqslant \sum_{j=1}^{d}\left\|D_{j} u-D_{j} P_{N} u\right\|_{m-1, \omega} \\
& \leqslant \sum_{j=1}^{d}\left\|D_{j} u-P_{N} D_{j} u\right\|_{m-1, \omega}+\sum_{j=1}^{d}\left\|P_{N} D_{j} u-D_{j} P_{N} u\right\|_{m-1, \omega} \\
& \leqslant C^{\prime} N^{e(m-1, \sigma-1)} \sum_{j=1}^{d}\left\|D_{j} u\right\|_{\sigma-1, \omega}+C^{\prime \prime} N^{e(m, \sigma)}\|u\|_{\sigma, \omega},
\end{aligned}
$$

applying the inductive hypothesis to each $D_{j} u \in H_{\omega}^{\sigma-1}(\Omega)$, and inequality (2.15). Whence the result, since $e(m-1, \sigma-1)<e(m, \sigma)$.

For any fixed $\mu$ and $\sigma$, the exponent $e(\mu, \sigma)$ in (2.16) is larger than the corresponding exponent in (1.1) for the Fourier system, whenever $\mu$ is different from zero (an increase of one in the order of derivatives on the left-hand side of (2.16) produces an increase of two in the power of $N$ on the right). According to the proof of the estimate, such a phenomenon is directly related to the inverse inequality (2.12), which in turn has been derived using the expression (2.10) for the first derivative in terms of the Chebyshev coefficients. Actually, this expansion is qualitatively quite different from the one in the Fourier system (recall that for basis functions one has $d \psi_{k} / d \theta=i k \psi_{k}$, hence $\left\|\psi_{k}\right\|_{1} \sim k$ in the Fourier system, while $d \phi_{k} / d x=k \sum_{l=0}^{\prime k-1} \phi_{l}$, hence $\left\|\phi_{k}\right\|_{1, w} \sim k \sqrt{k}$ in the Chebyshev system). A natural question arises, whether a bound better than (2.16) - with respect to $N$-may be derived. However, it is an easy matter to build up simple functions for which the value of $e(\mu, \sigma)$ for particular choices of $\mu$ and $\sigma$, cannot be diminished: for instance, the function

$$
u^{(N)}=\phi_{N+1}-(N+1) /(N-1) \phi_{N-1} \quad(N \text { odd })
$$

satisfies

$$
\left\|u^{(N)}-P_{N} u^{(N)}\right\|_{1, w} \sim \sqrt{N}\left\|u^{(N)}\right\|_{1, w}
$$


A different argument can be used in discussing the optimality of the bound (2.16); in order to explain it, we confine ourselves to the one-dimensional case, and for every $\mu$ and $\sigma$ (nonnegative integers) we denote by $\tilde{e}(\mu, \sigma)$ any real number such that the estimate

$$
\left\|u-P_{N} u\right\|_{\mu, w} \leqslant C N^{\tilde{e}(\mu, \sigma)}\|u\|_{\sigma, w} \quad \forall u \in H_{w}^{\sigma}(I),
$$

holds. Then

$$
\begin{aligned}
\left\|P_{N} D u-D P_{N} u\right\|_{m, w} & \leqslant\left\|D u-P_{N} D u\right\|_{m, w}+\left\|D u-D P_{N} u\right\|_{m, w} \\
& \leqslant C N^{\tilde{e}(m, s)}\|D u\|_{s, w}+C N^{\tilde{e}(m+1, s+1)}\|u\|_{s+1, w}
\end{aligned}
$$

On the other hand, (2.13) becomes in the present situation and for even $N$

$$
\hat{z}_{N} \phi_{0}^{(N)}+\hat{z}_{N+1} \phi 1^{(N)}=P_{N} D u-D P_{N} u
$$

if we set $D u=z=\sum_{k=0}^{N} \hat{z}_{k} \phi_{k}$. We note that $D^{j} \phi_{0}^{(N)}$ is orthogonal to $D^{j} \phi_{1}^{(N)}$ for any $j \geqslant 0$, since $\phi_{0}^{(N)}$ and $\phi_{1}^{(N)}$ are, respectively, an even and an odd function; moreover, by repeated application of formula $(2.10)$ one easily checks that

$$
\left\|D^{j} \phi_{0}^{(N)}\right\|_{0, w} \sim\left\|D^{j} \phi_{1}^{(N)}\right\|_{0, w} \sim N^{2 j+1 / 2}
$$

Hence

$$
\begin{aligned}
\left\|D^{m}\left(D P_{N} u-P_{N} D u\right)\right\|_{0, w}^{2} & =\left|\hat{z}_{N}\right|^{2}\left\|D^{m} \phi \delta^{N)}\right\|_{0, w}^{2}+\left|\hat{z}_{N+1}\right|^{2}\left\|\left.D^{m} \phi\right|^{(N)}\right\|_{0, w}^{2} \\
& \sim N^{4 m+1}\left(\left|\hat{z}_{N}\right|^{2}+\left|\hat{z}_{N+1}\right|^{2}\right) .
\end{aligned}
$$

Collecting (2.18) and (2.19), one has

$$
\left|\hat{z}_{N}\right| \leqslant C N^{\rho}\|z\|_{s, w} \quad \forall z \in H_{w}^{s}(I),
$$

where $\rho=\max (\tilde{e}(m+1, s+1), \tilde{e}(m, s))-2 m-1 / 2$. If one takes $\tilde{e}(\mu, \sigma)=$ $e(\mu, \sigma)$ defined by (2.17), then $\rho=-s$; conversely, if $\tilde{e}(\mu, \sigma)$ can be chosen strictly less than $e(\mu, \sigma)$, the exponent $\rho$ in (2.20) becomes strictly less than $-s$. Hence the optimality problem for $e(\mu, \sigma)$ in (2.16) has been reduced to the optimality problem for $\rho$ in (2.20).

A series of counterexamples can be built up in order to show that $\rho$ cannot be smaller than $-s$ in (2.20). We consider here the cases $s=0$ and $s=1$ :

(a) Case $s=0$. For each $\varepsilon>0$, define the sequence of positive integers $\{N(k)\}_{k=0}^{\infty}$ by the relation

$$
\left\{\begin{aligned}
N(0)= & 0 \\
N(k)= & \text { the smallest integer strictly larger than } N(k-1) \\
& \text { such that } N(k)^{e} \geqslant \pi k^{2} / \sqrt{6}
\end{aligned}\right.
$$

and set

$$
\hat{z}_{N}= \begin{cases}1 / k & \text { if } N=N(k) \text { for a } k \geqslant 1, \\ 0 & \text { otherwise. }\end{cases}
$$

Then $z=\sum_{N=0}^{\infty} \hat{z}_{N} \phi_{N}$ satisfies

$$
\|z\|_{0, w}^{2}=\sum_{k=0}^{\infty} \frac{1}{k^{2}}=\frac{\pi^{2}}{6} \quad \text { and } \quad\left|\hat{z}_{N(k)}\right| \geqslant k N(k)^{-\varepsilon}\|z\|_{0, w} .
$$


(b) Case $s=1$. Given $v=\sum_{N=0}^{\infty} \hat{v}_{N} \phi_{N}$, let $z=\sum_{N=0}^{\infty} \hat{z}_{N} \phi_{N}$ be a primitive of $v$. By (2.10) it is easily checked that

$$
\hat{z}_{N}=\frac{1}{2 N}\left\{\hat{v}_{N-1}-\hat{v}_{N+1}\right\}, \quad N \geqslant 1 \text {. }
$$

So, for any $\varepsilon>0$, define the sequence of positive integers $\{N(k)\}_{k=0}^{\infty}$ by the relation

$$
\left\{\begin{aligned}
N(0)= & 0 \\
N(k)= & \text { the smallest integer strictly larger than } N(k-1)+2 \\
& \text { such that } N(k)^{\varepsilon} \geqslant 2 \pi((1+\pi) / 6)^{1 / 2} \cdot k^{2}
\end{aligned}\right.
$$

and for any $N \geqslant 0$ set

$$
\hat{v}_{N}= \begin{cases}1 / k & \text { if } N=N(k)+1 \text { for a } k \geqslant 1, \\ 0 & \text { otherwise. }\end{cases}
$$

Then if $z$ is the primitive of $v$ which vanishes at the origin, one has

$$
\|z\|_{1, w} \leqslant \pi((1+\pi) / 6)^{1 / 2}
$$

so that

$$
\left|\hat{z}_{N(k)}\right|=\frac{1}{2 N(k)} \cdot \frac{1}{k} \geqslant k \cdot N(k)^{-(1+\varepsilon)}\|z\|_{1, w} .
$$

As a consequence of this discussion, we obtain the following result.

COROllary 2.1. There exists a function $u \in H_{w}^{1}(I)$ such that the sequence $\left\{P_{N} u\right\}_{N=0}^{\infty}$ is unbounded in $H_{w}^{1}(I)$.

Proof. Let $z=\sum_{N=0}^{\infty} \hat{z}_{N} \phi_{N} \in L_{w}^{2}(I)$ be such that

$$
\sqrt{N} \hat{z}_{N} \rightarrow \infty \text { as } N \rightarrow \infty \text {, }
$$

and define $u$ to be a primitive of $z$. Then, by (2.19) with $m=0$,

$$
\left\|P_{N} D u-D P_{N} u\right\|_{0, w} \rightarrow \infty \text { as } N \rightarrow \infty
$$

whence the result, since $\left\|P_{N} D u\right\|_{0, w} \leqslant\|z\|_{0, w}$.

2.2. The Legendre SPS. We choose $I=(-1,1)$ and $w(x) \equiv 1$. The orthogonal system associated with $w$ is $\left\{\phi_{k}=\lambda_{k} L_{k}\right\}_{k=0}^{\infty}$, where $L_{k}$ is the Legendre polynomial of degree $k$ normalized so that $L_{k}(1)=1$, and $\lambda_{k}=(k+1 / 2)^{1 / 2}$. The formal expansion of the first derivative of a function $v=\sum_{k=0}^{\infty} \hat{v}_{k} \phi_{k}$ is

$$
D v=2 \sum_{l=0}^{\infty} \lambda_{l}\left(\sum_{k=l+1}^{\infty} \lambda_{k} \hat{v}_{k}\right) \phi_{l} \text {. }
$$

Actually, this formula can be justified for every $v \in H^{1}(I)$ (see Proposition 2.1 below).

We first consider the $L^{2}$-norm of the approximation error $u-P_{N} u$.

THEOREM 2.3. For any real $\sigma \geqslant 0$, there exists a constant $C$ such that

$$
\left\|u-P_{N} u\right\|_{0} \leqslant C N^{-\sigma}\|u\|_{\sigma} \quad \forall u \in H^{\sigma}(\Omega) \text {. }
$$

Proof. Assume at first that $\sigma=2 p, p \geqslant 1$ integer. Define the differential operators in one space variable

$$
A_{j}=D_{j}\left(1-\left(x^{(j)}\right)^{2}\right) D_{j}
$$


and the following partition of the set $\mathscr{K}(N)=\left\{\left.\mathbf{k} \in \mathbf{N}^{d}|| \mathbf{k}\right|_{\infty}>N\right\}$ :

$$
\begin{aligned}
& \mathscr{K}^{(1)}(N)=\left\{\mathbf{k} \in \mathcal{K}(N) \mid k^{(1)}>N\right\}, \\
& \mathscr{K}^{(j)}(N)=\left\{\mathbf{k} \in \mathscr{K}(N) \backslash \bigcup_{l<j} \mathcal{K}^{(l)}(N) \mid k^{(j)}>N\right\}, \quad j=2, \ldots, d .
\end{aligned}
$$

For the moment, assume $u \in C^{\infty}(\bar{\Omega})$. For $\mathbf{k} \in \mathscr{K}^{(1)}(N)$ one has

$$
\begin{aligned}
\hat{u}_{\mathbf{k}} & =\left(u, \phi_{\mathbf{k}}\right)=-\frac{1}{k_{1}\left(k_{1}+1\right)} \int_{\Omega^{\prime}} \phi_{\mathbf{k}^{\prime}}\left(\mathbf{x}^{\prime}\right) d \mathbf{x}^{\prime} \int_{-1}^{1} u\left(x^{(1)}, \mathbf{x}^{\prime}\right) A_{1} \phi_{k_{1}}\left(x^{(1)}\right) d x^{(1)} \\
& =-\frac{1}{k_{1}\left(k_{1}+1\right)} \int_{\Omega^{\prime}} \phi_{\mathbf{k}^{\prime}}\left(\mathbf{x}^{\prime}\right) d \mathbf{x}^{\prime} \int_{-1}^{1} A_{1} u\left(x^{(1)}, \mathbf{x}^{\prime}\right) \phi_{k_{1}}\left(x^{(1)}\right) d x^{(1)} \\
& =-\frac{1}{k_{1}\left(k_{1}+1\right)}\left(A_{1} u, \phi_{\mathbf{k}}\right),
\end{aligned}
$$

where we have used the fact that $\phi_{k}$ satisfies the singular differential equation $D\left[\left(1-x^{2}\right) D \phi_{k}\right]+k(k+1) \phi_{k}=0$. By iteration we obtain

$$
\hat{u}_{\mathbf{k}}=\left[\frac{-1}{k_{1}\left(k_{1}+1\right)}\right]^{p}\left(A_{1}^{p} u, \phi_{\mathbf{k}}\right),
$$

hence, for arbitrary $j$,

$$
\begin{aligned}
\sum_{\mathbf{k} \in \mathcal{K}^{(\omega)}(N)}\left|\hat{u}_{\mathbf{k}}\right|^{2} & =\left(\frac{1}{k_{j}\left(k_{j}+1\right)}\right)^{2 p} \sum_{\mathbf{k} \in \mathcal{K}^{(N)}(N)}\left|\left(A_{j}^{p} u, \phi_{\mathbf{k}}\right)\right|^{2} \\
& \leqslant N^{-4 p}\left\|A_{j}^{p} u\right\|_{0}^{2}<C N^{-4 p}\|u\|_{2 p}^{2} .
\end{aligned}
$$

Since

$$
\left\|u-P_{N} u\right\|_{0}^{2}=\sum_{j=1}^{d} \sum_{k \in \mathscr{K}(N)(N)}\left|\hat{u}_{\mathbf{k}}\right|^{2},
$$

we obtain the result for $u$ regular, then for $u \in H^{2 p}(\Omega)$ by density and continuity. Finally, for $\sigma$ included between two even integers, we conclude by interpolation (see Bergh and Löfström [4, Theorem 3.1.2]).

The estimates for higher order Sobolev norms of the error can be derived by the same arguments we used in the Chebyshev case. Actually, the formal expansion of the first derivative (2.21) exhibits the same structure, hence the same asymptotic behavior, both for the Chebyshev and for the Legendre system. So, we simply recall the main results.

LEMMA 2.4 (inverse inequality). For any real $\mu$ and $\nu$ such that $0<\nu<\mu$, one has

$$
\|u\|_{\mu} \leqslant C N^{2(\mu-\nu)}\|u\|_{\nu} \quad \forall u \in S_{N} .
$$

(This inequality has been also obtained by Babuška, Szabö and Katz [3] by a different technique.)

THEOREM 2.4. For any real $\mu$ and $\sigma$ such that $0 \leqslant \mu<\sigma$, there exists a constant $C$ such that

$$
\left\|u-P_{N} u\right\|_{\mu}<C N^{e(\mu, \sigma)}\|u\|_{\sigma} \quad \forall u \in H^{\sigma}(\Omega)
$$

where $e(\mu, \sigma)$ is given by (2.17). 
Before the end of this section, we will show that expansion (2.21) is justified for every $v \in H^{1}(I)$.

Proposition 2.1. Assume $v=\sum_{k=0}^{\infty} \hat{v}_{k} \phi_{k} \in H^{1}(I)$, and set $D v=\sum_{l=0}^{\infty} \hat{z}_{l} \phi_{l}$. Then

$$
\hat{z}_{l}=2 \lambda_{l} \sum_{k=l+1}^{\infty} \lambda_{k} \hat{v}_{k}, \quad l=0,1, \ldots
$$

Proof. Set $D P_{N} v=\sum_{l=0}^{N-1} z_{l}^{(N)} \phi_{l}$. By (2.21), $z_{l}^{(N)}=2 \lambda_{l} \sum_{k=l+1}^{N} \lambda_{k} \hat{v}_{k}$, hence (2.25) is equivalent to

$$
\left(D P_{N} v, \phi_{l}\right) \rightarrow\left(D v, \phi_{l}\right) \text { as } N \rightarrow \infty, l=0,1, \ldots
$$

Note that, due to the analogy of Corollary 2.1 for the Legendre system, the sequence $\left\{P_{N} v\right\}_{N=0}^{\infty}$ may not be $H^{1}$-weakly convergent to $v$; however, estimate (2.24) with $\mu=1 / 2+\varepsilon$ and $s=1$, plus the Sobolev imbedding theorem, yield

$$
\left\|v-P_{N} v\right\|_{L^{\infty}(I)} \leqslant C N^{-1 / 4+\varepsilon}\|v\|_{1}, \quad \varepsilon>0 \text { arbitrary. }
$$

Since

$$
\left(D P_{N} v, \phi_{l}\right)=-\left(P_{N} v, D \phi_{l}\right)+\left[\left(P_{N} v\right) \phi_{l}\right]_{x= \pm 1},
$$

we can pass to the limit and obtain the result.

Remark 2.2. Let us consider, for example, a Chebyshev or Legendre spectral (Galerkin) approximation to the Laplace or to the heat equation; see [10]. Standard energy estimates give an upper bound of the approximation error in the norm of $H_{\omega}^{1}(\Omega)$ in terms of $\left\|u-P_{N} u\right\|_{1, \omega}$. Using directly the estimate (2.16) or (2.24) we get the suboptimal order of convergence $O\left(N^{3 / 2-\sigma}\right)$ assuming $u \in H_{\omega}^{\sigma}(\Omega)$. So the results of this section indicate that projection operators for higher order norms must be involved in the analysis of nonperiodic problems (in opposition to the case of periodic problems, where the projector $P_{N}$ plays a universal rôle). Actually, using the $H_{\omega}^{1}$-projection operator $\Pi_{N}$, we obtain $O\left(N^{1-\sigma}\right)$. A similar result also holds for the Chebyshev (Legendre) approximation to the steady state Burgers equation, as shown in [22, Section 2].

The proof of the estimate $\left\|u-\Pi_{N} u\right\|_{\mu, \omega} \leqslant C N^{\mu-\sigma}\|u\|_{\sigma, \omega}$, which holds for $u \in$ $H_{\omega}^{\sigma}(\Omega), 0 \leqslant \mu \leqslant \sigma>1$, relies on the properties (2.20) and (2.32) for $\mu=0$; cf. [22, Theorem 1.6].

The property (2.24) is also used to analyze a Legendre spectral approximation to the one-dimensional advection equation; see [11, Section 3.2]. Furthermore, the estimates (2.16) and (2.24) are used to get the results (3.7) and (3.15) which play an important role in the applications (see Remark 3.2).

3. Spectral Interpolation Systems. The motivation for the results of this section stands in the analysis of pseudo-spectral (collocation) approximations of P.D.E.'s submitted to nonperiodic boundary conditions in a ipercube. The strategy to be followed is to collocate the equation at the interior knots of some Gauss integration formulas having also knots where boundary conditions are imposed. So, for elliptic and parabolic problems one uses totally closed (Gauss-Lobatto) quadrature. For first order hyperbolics, open or semiopen (Gauss or Gauss-Radau) quadrature is also needed, according to the direction of the flux. Interpolation operators at the quadrature nodes are considered in the analysis of such schemes. 
In the following, an integration formula on $I$

$$
\int_{I} \phi(x) w(x) d x \simeq \sum_{m=0}^{N} \phi\left(x_{m}\right) w_{m}, \quad x_{0}<x_{1}<\cdots<x_{N},
$$

will be denoted by $f_{w, N}=\left\{\left(x_{m}, w_{m}\right) \mid 0 \leqslant m \leqslant N\right\}$. We fix a finite set $\mathscr{F}_{w, N}$ of such formulas, and we assume that each $f_{w, N} \in \mathscr{F}_{w, N}$ satisfies

(i) $\quad x_{m} \in \bar{I}, w_{m}>0$, for $0 \leqslant m \leqslant N$;

(ii) the formula is exact if $\phi$ is a polynomial of degree $\leqslant 2 N-1$.

To every $f_{w, N}$, we associate the symmetric bilinear continuous form on $C^{0}(\bar{I})$

$$
(u, v)_{w, N}=\sum_{m=0}^{N} u\left(x_{m}\right) v\left(x_{m}\right) w_{m}
$$

due to (3.2), it defines an inner product on $S_{N}(I)$, with respect to which the system $\left\{\phi_{k}\right\}_{k=0}^{N}$ satisfies

$$
\left(\phi_{l}, \phi_{k}\right)_{w, N}= \begin{cases}0, & \text { if } l \neq k, l, k \leqslant N \\ 1, & \text { if } l=k<N \\ e_{N}, & \text { if } l=k=N\end{cases}
$$

with $e_{N}>0$ depending on $f_{w, N}$.

In this paper we take $\mathscr{F}_{w, N}=\left\{f_{w, N}^{(G)}, f_{w, N}^{(G R)}, f_{w, N}^{(G L)}\right\}$, where $f_{w, N}^{(G)}, f_{w, N}^{(G R)}, f_{w, N}^{(G L)}$ are, respectively, the $(N+1)$-point Gauss $\left(e_{N}=1,-1<x_{0}<x_{N}<1\right)$, Gauss-Radau $\left(e_{N}=1,-1=x_{0}<x_{N}<1\right)$, Gauss-Lobatto $\left(e_{N} \neq 1,-1<x_{0}<x_{N}=1\right)$ integration formulas over $I$ with respect to the weight $w$. For $d \geqslant 1$, a $d$-tuple $f_{\omega, N}=$ $\left(f_{w, N}^{(j)}\right)_{j=1}^{d} \in\left(\mathscr{F}_{w, N}\right)^{d}$ of one-dimensional integration formulas defines the integration formula on $\Omega$

$$
\int_{\Omega} \phi(\mathbf{x}) \omega(\mathbf{x}) d \mathbf{x} \simeq \sum_{\substack{\mathbf{m} \in \mathbf{N}^{d} \\|\mathbf{m}|_{\infty}<N}} \phi\left(\mathbf{x}_{\mathbf{m}}\right) \omega_{\mathbf{m}}
$$

where $\mathbf{x}_{\mathbf{m}}=\left(x_{m}^{(j)}\right)_{j=1}^{d}, \omega_{\mathbf{m}}=\Pi_{j=1}^{d} w_{m_{j}}^{(j)}\left(\right.$ if $\left.f_{w, N}^{(j)}=\left\{\left(x_{m}^{(j)}, w_{m}^{(j)}\right) \mid 0 \leqslant m \leqslant N\right\}\right)$. We introduce the bilinear form on $C^{0}(\bar{\Omega})$

$$
(u, v)_{\omega, N}=\sum_{\substack{\mathbf{m} \in \mathbf{N}^{d} \\|\mathbf{m}|_{\infty}<N}} u\left(\mathbf{x}_{\mathbf{m}}\right) v\left(\mathbf{x}_{\mathbf{m}}\right) \omega_{\mathbf{m}}
$$

and we note that

$$
\left(\phi_{\mathbf{k}}, \phi_{1}\right)_{\omega, N}=\prod_{j=1}^{d}\left(\phi_{k_{j}}, \phi_{l_{j}}\right)_{w, N}^{(j)},
$$

where the superscript $j$ refers to the formula $f_{w, N}^{(j)}$. In particular, (3.4) is an inner product on $S_{N}$; hence we can define the continuous linear operator $P_{c}: C^{0}(\bar{\Omega}) \rightarrow S_{N}$ by the relation

$$
\left(P_{c} v, \phi\right)_{\omega, N}=(v, \phi)_{\omega, N} \quad \forall \phi \in S_{N} .
$$

Since for every $\mathbf{x}_{\mathbf{m}}$ there exists a unique $\phi \in S_{N}$ satisfying $\phi\left(\mathbf{x}_{\mathrm{l}}\right)=\delta_{\mathrm{m}, \mathrm{l}}$ (precisely $\phi=\Pi_{j=1}^{d} \phi^{(j)}$, with $\left.\phi^{(j)}\left(x_{l}^{(j)}\right)=\delta_{m_{j}, l}\right)$, we see that $P_{c} v$ is the function in $S_{N}$ which interpolates $v$ at the nodes of the integration formula $f_{\omega, N}$. 
Definition 3.1. The triplet $\left(\Omega, f_{\omega, N}, P_{c}\right)$ is called a Spectral Interpolation System (SIS).

3.1. The Chebyshev SIS. The quadrature nodes (Chebyshev points) are of the form $x_{m}=\cos \theta_{m}, 0 \leqslant m \leqslant N$. For the Gauss formula, $\theta_{m}$ are defined in (1.4), (1.8) and $w_{m}=\pi /(N+1)$ for any $m$. For the Gauss-Radau formula, $\theta_{m}$ are defined in (1.4), (1.11) and $w_{0}=\pi /(2 N+1), w_{m}=2 \pi /(2 N+1)$ for $m \geqslant 1$. For the GaussLobatto formula, $\theta_{m}$ are defined in (1.4), (1.14), $w_{0}=w_{N}=\pi / 2 N, w_{m}=\pi / N$ for $1 \leqslant m \leqslant N-1$, and $e_{N}=2$.

Moreover we can associate to the interpolation operator $\boldsymbol{P}_{c}$ over $\Omega$ a Fourier interpolation operator $\tilde{\Pi}_{c}$ over $\tilde{\Omega}$. Precisely, we define the partition $\{J(G), J(G R), J(G L)\}$ of $\mathscr{D}$ by requiring that $j \in J(G)$ (resp. $J(G R)$, resp. $J(G L))$ whenever $f_{w, N}^{(j)}$ is an integration formula of Gauss (resp. Gauss-Radau, resp. Gauss-Lobatto) type; then we define $\tilde{\Pi}_{c}$ by (1.22).

LEMMA 3.1. For any $v \in C^{0}(\bar{\Omega})$

$$
\widetilde{P_{c} v}=\tilde{\Pi}_{c} \tilde{v}
$$

Proof. By the relation $\cos k \theta=(\exp (i k \theta)+\exp (-i k \theta)) / 2$ and the fact that $P_{c} v \in S_{N}$, one easily checks that $\widetilde{P_{c} v} \in \tilde{\Xi}_{N}(\tilde{\Omega})$. Moreover by definition $\widetilde{P_{c} v}$ agrees with $\tilde{v}$ at the nodes $\boldsymbol{\theta}_{m}$ with $0 \leqslant m_{j} \leqslant N$ for any $j \in \mathscr{D}$. Since the interpolation nodes of type $(G),(G R)$ or $(G L)$ are symmetrically placed with respect to the origin, and $\widetilde{P_{c} v}, \tilde{v}$ are both even functions in each coordinate, they agree at any node $\boldsymbol{\theta}_{\mathbf{m}}, \mathbf{m} \in \zeta(N)$, whence the result by the uniqueness of the interpolation operator.

THEOREM 3.1. For any $\sigma>d / 2$ and any real $\mu$ such that $0 \leqslant \mu \leqslant \sigma$, there exists $a$ constant $C$ such that

$$
\left\|u-P_{c} u\right\|_{\mu, \omega} \leqslant C N^{2 \mu-\sigma}\|u\|_{\sigma, \omega} \quad \forall u \in H_{\omega}^{\sigma}(\Omega) .
$$

Proof. One has

$$
\left\|u-P_{c} u\right\|_{0, \omega}^{2}=2^{-d}\left\|\tilde{u}-\tilde{\Pi}_{c} \tilde{u}\right\|_{L^{2}(\tilde{\Omega})}^{2}
$$

so (3.7) holds with $\mu=0$ by (1.23) and (2.7). For $\mu>0$, we use the inverse inequality (2.11)

$$
\left\|u-P_{c} u\right\|_{\mu, \omega} \leqslant\left\|u-P_{N} u\right\|_{\mu, \omega}+C N^{2 \mu}\left\|P_{N} u-P_{c} u\right\|_{0, \omega},
$$

and we conclude by (2.16) and the previous case.

3.2. The Legendre SIS.

LEMMA 3.2. Let $e_{N}$ be defined by (3.3) for the Gauss-Lobatto integration formula $f_{w, N}^{(G L)}$. Then

$$
e_{N}=2+1 / N \text {. }
$$

Proof. Since $f_{w, N}^{(G L)}$ is exact for polynomials of degree $\leqslant 2 N-1$, we have

$$
\left(\phi_{N}, \phi_{N}\right)_{N}-\left(\phi_{N}, \phi_{N}\right)=\alpha_{N}^{2}\left[\left(x^{N}, x^{N}\right)_{N}-\left(x^{N}, x^{N}\right)\right]
$$

where

$$
\alpha_{N}=\lambda_{N} \frac{1}{N ! 2^{N}} \cdot \frac{(2 N) !}{N !}
$$


is the leading coefficient of the polynomial $\phi_{N}$. By the formula for the GaussLobatto integration error (cf., e.g., Davis and Rabinowitz [12, p. 80]) we get

$$
\left(x^{N}, x^{N}\right)_{N}-\left(x^{N}, x^{N}\right)=\frac{(N+1) N^{3} 2^{2 N+1}[(N-1) !]^{4}}{(2 N+1)[(2 N) !]^{3}} \frac{d^{2 N}}{d x^{2 N}}\left(x^{2 N}\right),
$$

whence the result can be easily deduced.

As a consequence we note that, due to (3.5), we have

$$
\|\phi\|_{0}^{2} \leqslant(\phi, \phi)_{N} \leqslant C\|\phi\|_{0}^{2} \quad \forall \phi \in S_{N}(\Omega),
$$

with $C$ independent of $N$.

Before stating the error estimate for the interpolation operator $P_{c}$, we derive the following auxiliary result.

Lemma 3.3. Assume $d=1$. For any $0<s<1$ there exists a projection operator

$$
\Pi_{s, N}: H^{s}(I) \rightarrow S_{N}
$$

such that the following estimate holds for any $u \in H^{\circ}(I)$ :

$$
\left\|u-\Pi_{s, N} u\right\|_{\mu} \leqslant C N^{\mu-\sigma}\|u\|_{\sigma}, \quad 0 \leqslant \mu \leqslant s \leqslant \sigma .
$$

$\Pi_{s, N}$ is the orthogonal projection operator with respect to a suitable inner product, whose associate norm is equivalent to the standard $H^{s}$-norm.

Proof. By an affine mapping we can transform the interval $I$ into $(0, \pi)$, without affecting the result; so assume we work on $(0, \pi)$. For any $v \in H^{s}(0, \pi)$, denote by $R v$ the function obtained by even reflection with respect to the origin. Since $s<3 / 2, R v \in H_{p}^{s}(-\pi, \pi)$. This space will be equipped with the inner product

$$
((U, V))_{H^{s}(-\pi, \pi)}=\sum_{k=-\infty}^{+\infty}\left(1+k^{2}\right)^{s} \hat{u}_{k} \overline{\hat{v}}_{k}
$$

if $U=\sum_{k=-\infty}^{+\infty} \hat{u}_{k} \psi_{k}, V=\sum_{k=-\infty}^{+\infty} \hat{v}_{k} \psi_{k}$. So we define the following inner product in $H^{s}(0, \pi)$

$$
((u, v))_{H^{s}(0, \pi)}=\frac{1}{2}((R u, R v))_{H^{s}(-\pi, \pi)}
$$

and $\Pi_{s, N}$ will be the orthogonal projection operator upon $S_{N}$ with respect to this inner product. It is easily checked that $u \rightarrow((u, u))_{H^{3}(0, \pi)}^{1 / 2}$ is a norm equivalent to $\|\cdot\|_{s}$. In order to establish (3.10), consider first the case $\mu=s$. Since $\Pi_{s, N}$ is an orthogonal projection, we have

$$
\left\|u-\Pi_{s, N} u\right\|_{s} \leqslant C\|u\|_{s} \quad \forall u \in H^{s}(0, \pi) .
$$

Moreover, for $\sigma \geqslant 1$ denote by $\Pi_{1, N}: H^{1}(0, \pi) \rightarrow S_{N}$ the orthogonal projection in the $H^{1}$-norm; then

$$
\left\|u-\Pi_{s, N} u\right\|_{s} \leqslant C\left\|u-\Pi_{1, N} u\right\|_{s} \leqslant C N^{s-\sigma}\|u\|_{\sigma} \quad \forall u \in H^{\sigma}(0, \pi)
$$

(cf. Maday and Quarteroni [22]). When $s \leqslant \sigma \leqslant 1$, we use interpolation taking into account (3.11) and (3.12).

We consider now the case $\mu=0$, arguing by a standard duality argument. Denote by

$$
J_{s}:\left(H^{s}(0, \pi)\right)^{\prime} \rightarrow H^{s}(0, \pi)
$$


the Riesz isomorphism (i.e., $J_{s}$ satisfies

$$
\left.\left(\left(J_{s} f, v\right)\right)_{H^{s}(0, \pi)}={ }_{\left(H^{s}(0, \pi)\right)^{\prime}}\langle f, v\rangle_{H^{s}(0, \pi)} \quad \forall v \in H^{s}(0, \pi)\right)
$$

and assume for the moment that the following regularity result holds:

$$
J_{s} \text { maps } L^{2}(0, \pi) \text { into } H^{2 s}(0, \pi) \text { with continuity. }
$$

Then

$$
\begin{aligned}
\left\|u-\Pi_{s, N} u\right\|_{0} & =\sup _{\substack{f \in L^{2}(0, \pi) \\
\|f\|_{0}=1}}\left(u-\Pi_{s, N} u, f\right) \\
& =\sup _{\substack{f \in L^{2}(0, \pi) \\
\|f\|_{0}=1}}\left(\left(u-\Pi_{s, N} u, J_{s} f\right)\right)_{H^{s}(0, \pi)} \\
& =\sup _{\substack{f \in L^{2}(0, \pi) \\
\|f\|_{0}=1}}\left(\left(u-\Pi_{s, N} u, J_{s} f-\Pi_{s, N} J_{s} f\right)\right)_{H^{s}(0, \pi)} \\
& \leqslant C N^{s-\sigma}\|u\|_{\sigma} \cdot N^{-s} \sup _{\substack{f \in L^{2}(0, \pi) \\
\|f\|_{0}=1}}\left\|J_{s} f\right\|_{2 s} \leqslant C N^{-\sigma}\|u\|_{\sigma}
\end{aligned}
$$

due to (3.13), to (3.10) with $\mu=s$ and to (3.14). Finally we obtain (3.10) with $0<\mu<s$ by interpolation. So the proof is complete if we check (3.14). To this end, set $R J_{s} f=\sum_{k} \hat{w}_{k} \psi_{k}, R v=\Sigma_{k} \hat{v}_{k} \psi_{k}, R f=\Sigma_{k} \hat{f}_{k} \psi_{k}$. Equation (3.13) is equivalent to

$$
\left(\left(R J_{s} f, R v\right)\right)_{H^{s}(-\pi, \pi)}=(R f, R v)_{L^{2}(-\pi, \pi)} \quad \forall v \in H^{s}(0, \pi) \text {, }
$$

that is

$$
\sum_{k}\left(1+k^{2}\right)^{s} \hat{w}_{k} \hat{v}_{k}=\sum_{k} \hat{f}_{k} \hat{v}_{k} \quad \forall v \in H^{s}(0, \pi)
$$

Note that the Fourier coefficients involved in this equation are all real and even, i.e., $\hat{w}_{k}=\hat{w}_{-k}, \hat{v}_{k}=\hat{v}_{-k}, \hat{f}_{k}=\hat{f}_{-k}$ for any $k \in \mathbf{Z}$. Taking $v(x)=\cos k x$, we get

$$
\left(1+k^{2}\right)^{s} \hat{w}_{k}=\hat{f}_{k}, \quad k \in \mathbf{Z}
$$

whence $R J_{s} f \in H^{2 s}(-\pi, \pi)$ with

$$
\left(\left(R J_{s} f, R J_{s} f\right)\right)_{H^{2 s}(-\pi, \pi)}=(R f, R f)_{L^{2}(-\pi, \pi)} .
$$

This clearly implies (3.14), and the proof is finished.

THEOREM 3.2. For any real $\sigma>d / 2$ and any real $\mu$ such that $0 \leqslant \mu \leqslant \sigma$, there exists a constant $C$ such that

$$
\left\|u-P_{c} u\right\|_{\mu} \leqslant C N^{2 \mu-\sigma+d-1 / 2}\|u\|_{\sigma} \quad \forall u \in H^{\sigma}(\Omega) .
$$

Proof. We first consider the case $\mu=0$. Denote by $\Pi: H^{\sigma}(\Omega) \rightarrow S_{N}$ a projection operator to be specified in the following. We write

$$
u-P_{c} u=(u-\Pi u)-P_{c}(u-\Pi u),
$$

and we estimate the second term on the right. We have

$$
\begin{aligned}
\left\|P_{c}(u-\Pi u)\right\|_{0}^{2} & \leqslant\left(P_{c}(u-\Pi u), P_{c}(u-\Pi u)\right)_{N} \text { by }(3.9) \\
& =(u-\Pi u, u-\Pi u)_{N} \\
& \leqslant 2^{d}\|u-\Pi u\|_{L^{\infty}(\Omega)}^{2} \cdot\left(\phi_{0}, \phi_{0}\right)_{N} \leqslant C\|u-\Pi u\|_{L^{\infty}(\Omega)}^{2} .
\end{aligned}
$$


Let us denote by $B_{p q}^{s}(\Omega)$ the Besov space of order $s$ and indices $p, q$ (see, e.g., Bergh and Löfström [4, Definition 6.22]). Since $B_{2}^{d}{ }^{2} \subseteq L^{\infty}(\Omega)$ with continuous injection (Bergh and Löfström [4, Chapter 6, Exercise 9]) and for any $\varepsilon>0, B_{21}^{d / 2}=$ $\left(H^{d / 2+\varepsilon}(\Omega), H^{d / 2-\varepsilon}(\Omega)\right)_{1 / 2,1}$ (where $(,)_{p, q}$ denotes here real interpolation of indices $p$ and $q$; see Bergh and Löfström [4, Theorem 6.2.4]) we have

$$
\begin{aligned}
\|u-\Pi u\|_{L^{\infty}(\Omega)}^{2} & \leqslant C_{1}\|u-\Pi u\|_{B_{2 / 2}^{d / 2}(\Omega)}^{2} \\
& \leqslant C_{2}\|u-\Pi u\|_{H^{d / 2+e}(\Omega)}\|u-\Pi u\|_{H^{d / 2-e(\Omega)},}
\end{aligned}
$$

where $C_{2}$ can be chosen independent of $\varepsilon$. Now we choose $\Pi=\Pi_{1 / 2+\varepsilon, N}$ defined in Lemma 3.3 when $d=1$, and $\Pi=P_{N}$ when $d>1$. Using (3.10) or (2.32) and letting $\varepsilon$ tend to zero, we obtain the result. For $\mu>0$ we proceed as in the proof of Theorem 3.1.

Remark 3.1. It is an open problem to check whether the last result is optimal.

Remark 3.2. Results (3.7) and (3.15) are currently used whenever Chebyshev and Legendre pseudo-spectral (collocation) methods are analyzed. The reader interested in applications can refer to [11] and [23] (concerned with the one-dimensional advection equation, [20] (heat equation), [21] (steady-state Burgers equation), where error estimates for the collocation approximation are derived by variational techniques. In particular one has to evaluate terms as $u-P_{c} u$ and $E_{\omega}(f, \phi)=(f, \phi)_{\omega, N}$ $-(f, \phi)_{\omega}$ for some continuous $f$ and $\phi \in S_{N}$. The last quantity defines the error arising from the use of an integration formula to approximate the $L_{\omega}^{2}$-inner product $(\cdot, \cdot)_{\omega}$. It can be proved $[10$, Lemma 3.2] that

$$
\left|E_{\omega}(f, \phi)\right| \leqslant C\|\Phi\|_{0, \omega}\left(\left\|f-P_{c} f\right\|_{0, \omega}+\left\|f-P_{N-1} f\right\|_{0, \omega}\right),
$$

where $C$ does not depend on $N$. Then, using (2.16), (2.24), (3.7) and (3.15), it follows that the above error behaves as $N^{-\sigma}$ assuming $f \in H_{\omega}^{\sigma}$.

Istituto di Matematica Applicata

Piazza L. Da Vinci

27100 Pavia, Italy

Istituto di Analisi Numerica del C.N.R.

Corso Carlo Alberto, 5

27100 Pavia, Italy

1. R. A. Adams, Sobolev Spaces, Academic Press, New York, 1975.

2. A. Avantaggiati, "Spazi di Sobolev con peso ed alcune applicazioni," Boll. Un. Mat. Ital., v. 5, 1976, pp. 1-52.

3. I. BABuŠKa, B. A. SzABö \& I. N. KaTZ, The P-Version of the Finite Element Method, Report of the Washington University, St. Louis, 1979.

4. J. Bergh \& J. LÖFSTRÖM, Interpolation Spaces. An Introduction, Springer-Verlag, Berlin and New York, 1976.

5. K. P. BUBE, “ $C^{m}$ convergence of trigonometric interpolants," SIAM J. Numer. Anal., v. 15, 1978, pp. $1258-1268$.

6. P. L. BUTZER \& H. Berens, Semigroups of Operators and Approximation, Springer-Verlag, Berlin and New York, 1967.

7. P. L. Butzer \& R. L. Nessel, Fourier Analysis and Approximation, Vol. 1, Birkhäuser-Verlag, Basel-Stuttgart, 1971.

8. A. P. Calderon, "Intermediate spaces and interpolation, the complex method," Studia Math., v. 24, 1964, pp. 113-190

9. C. Canuto \& A. Quarteroni, "Propriétés d'approximation dans les espaces de Sobolev de systèmes de polynômes orthogonaux," C. R. Acad. Sci. Paris. Sér. A-B, v. 290, 1980, pp. A925-A928. 
10. C. Canuto \& A. QuARTERoni, "Spectral and pseudo-spectral methods for parabolic problems with non periodic boundary conditions," Calcolo. (To appear.)

11. C. Canuto \& A. QuARTERon, "Error estimates for spectral and pseudo-spectral approximations of hyperbolic equations," SIAM J. Numer. Anal. (To appear.)

12. P. J. Davis \& P. Rabinowitz, Methods of Numerical Integration, Academic Press, New York, 1975.

13. D. Gottleb \& S. A. Orszag, Numerical Analysis of Spectral Methods: Theory and Applications, Regional Conf. Series in Appl. Math., SIAM, Philadelphia, Pa., 1977.

14. H. O. Kreiss \& J. Oliger, "Comparison of accurate methods for the integration of hyperbolic equations," Tellus, v. 24, 1972, pp. 199-215.

15. H. O. Kreiss \& J. Oliger, "Stability of the Fourier method," SIAM J. Numer. Anal., v. 16, 1979, pp. 421-433.

16. A. KufNer, O. John \& S. FucIK, Function Spaces, Noordhoff, Leyden, 1977.

17. J. L. Lions, "Théorèmes de traces et d'interpolation (I) ... (V)," (I), (II), Ann. Sc. Norm. Sup. Pisa, v. 13, 1959, pp. 389-403; v. 14, 1960, pp. 317-331; (III) J. Liouville, v. 42, 1963, pp. 196-203; (IV) Math. Ann., v. 151, 1963, pp. 42-56; (V) An. Acad. Brasil. Ciênc., v. 35, 1963, pp. 1-110.

18. J. L. Lions \& E. MAgenes, Non Homogeneous Boundary Value Problems and Applications, Vol. 1, Springer-Verlag, Berlin and New York, 1972.

19. J. L. Lions \& J. Peetre, “Sur une classe d'espaces d'interpolation," Inst. Hautes Études Sci. Publ. Math., v. 19, 1964, pp. 5-68.

20. Y. MADAY \& A. QUARTERONI, "Spectral and pseudo-spectral approximations of Navier-Stokes equations," SIAM J. Numer. Anal. (To appear.)

21. Y. MADAY \& A. QUARTERONI, “Approximation of Burgers' equation by pseudo-spectral methods," Math. Comp. (To appear.)

22. Y. MADAY \& A. QUARTERoni, “Legendre and Chebyshev spectral approximations of Burgers' equation," Numer. Math., v. 37, 1981, pp. 321-332.

23. B. MerCIER, "Stabilité et convergence des méthodes spectrales pour des problèmes d'évolution linéaires non périodiques. Application à l'équation d'advection," R.A.I.R.O. Anal. Numér. (To appear).

24. S. M. Nikol'SkII, Approximation of Functions of Several Variables and Imbedding Theorems, Springer-Verlag, Berlin and New York, 1975.

25. S. A. OrszaG, "Numerical simulation of incompressible flows within simple boundaries: IGalerkin (spectral) representations," Stud. Appl. Math., v. 2, 1971, pp. 293-326.

26. S. A. Orszag, "Numerical simulation of incompressible flows within simple boundaries: II-Accuracy,” J. Fluid Mech., v. 49, 1971, pp. 75-112.

27. J. PASCiAK, "Spectral and pseudo-spectral methods for advection equations," Math. Comp., v. 35, 1980, pp. $1081-1092$.

28. J. PeEtre, "Interpolation functions and Banach couples," Actes Congrès Internat. Math., 1970, v. 2, 1971, pp. 173-190.

29. G. SANSONE, Orthogonal Functions, Interscience, New York, 1959.

30. G. Szegö, Orthogonal Polynomials, Amer. Math. Soc. Colloq. Publ., Vol. 23, Amer. Math. Soc., Providence, R. I., 1939.

31. H. TRIEBEL, Interpolation Theory, Function Spaces, Differential Operators, North-Holland, Amsterdam, 1978.

32. A. Zygmund, Trigonometric Series,, Vol. 1, Cambridge Univ. Press, Cambridge, 1968. 